\title{
TSPAN1 upregulates MMP2 to promote pancreatic cancer cell migration and invasion via PLC $\gamma$
}

\author{
XIAOBO ZHANG ${ }^{1}$, GANG SHI $^{2}$, FENG GAO $^{1}$, PENG LIU $^{1}$, HUAITAO WANG ${ }^{1}$ and XIAODONG TAN ${ }^{1}$ \\ ${ }^{1}$ Department of General Surgery, Shengjing Hospital of China Medical University; \\ ${ }^{2}$ Department of Colorectal Surgery, Cancer Hospital of China Medical University, Liaoning Cancer Hospital and Institute, \\ Shenyang, Liaoning 110042, P.R. China
}

Received August 2, 2018; Accepted January 25, 2019

DOI: $10.3892 /$ or.2019.6989

\begin{abstract}
Pancreatic cancer (PC), a malignancy of the digestive system, has one of the highest rates of metastasis and mortality. It is characterized by the detachment, migration, implantation and infiltration of tumor cells to form metastases or recurrent foci. Tetraspanin 1 (TSPAN1), a novel member of the TSPAN superfamily, is highly expressed in many types of cancer, including gastric, colon, liver and esophageal cancer. It has also been associated with lymph node metastasis, tumor recurrence and metastasis. However, the role of TSPAN1 in PC has not been fully elucidated. The aim of the present study was to determine the expression of TSPAN1 in human PC tissue samples and cell lines. Additionally, the functions of TSPAN1 in PC cell migration and invasion were assessed. The protein and gene expression of TSPAN1 was analyzed in clinical PC tissue samples and human PC cell lines (SW1990, BxPC3, Capan1 and PANC-1) via immunohistochemistry, reverse transcription-quantitative polymerase chain reaction and western blotting. The effect of TSPAN1 downregulation and overexpression in PC cells, via transfection with siRNA and pLNCX-TSPAN1-cDNA recombinant plasmid, respectively, on cell invasion and migration were assessed. Additionally, the mRNA expression of matrix metalloproteinase (MMP2) and MMP9 were determined. In clinical PC tissue samples, the expression of TSPAN1 was markedly increased when compared with normal pancreatic tissue samples. TSPAN1 was also highly expressed in PC cell lines compared with HPDE, a normal pancreatic cell line. Transfection with siRNA targeting TSPAN1 in PC cell lines significantly suppressed PC cell migration and invasion, and downregulated the expression of MMP2. However, there was no effect on MMP9.
\end{abstract}

Correspondence to: Professor Xiaodong Tan, Department of General Surgery, Shengjing Hospital of China Medical University, 36 Sanhao Street, Heping, Shenyang, Liaoning 110042, P.R. China E-mail: tanxdcmu@163.com

Key words: human pancreatic cancer cells, tetraspanin 1, cell migration, cell invasion, matrix metalloproteinase 2, phospholipase $\mathrm{C} \gamma$
Consistently, PC cell migration and invasion together with MMP2 mRNA expression were markedly increased following TSPAN1 ectopic overexpression. The present study utilized small interfering RNAs (siRNA) targeted to phospholipase $\mathrm{C} \gamma$ (PLC $\gamma$ ) to demonstrate that TSPAN1 siRNA suppressed PC cell migration and invasion, and MMP2 mRNA expression by blocking the translocation and phosphorylation of PLC $\gamma$. The results of the present study revealed that TSPAN1 has an important function in human PC cell migration and invasion by modulating MMP2 expression via PLC $\gamma$. Thus, the results indicate that the silencing of TSPAN1 may be a potential therapeutic target for the treatment of PC.

\section{Introduction}

Pancreatic cancer (PC) has one of the highest mortality rates among all tumor-associated diseases (1). Less than one-fifth of patients with PC survive the first year, with a 5-year survival rate $<6 \%(1,2)$. The majority of patients with $\mathrm{PC}$ are diagnosed at a late stage and succumb due to the invasion and migration of cancer cells $(3,4)$. Current treatment methods, including surgical resection, radiation and chemotherapy do not significantly increase patient long-term survival (5-8). However, advancements in molecular biological techniques have created an opportunity for the exploration of effective targeted therapies for the treatment of PC.

Tetraspanins (TSPANs), also known as transmembrane 4 superfamily (TM4SF) proteins, is composed of a group of heterogeneous adaptor proteins, which exist in the form of TSPAN-enriched microdomains $(9,10)$. As its name indicates, TM4SF consists of four transmembrane domains that interact with various cell surface signaling molecules, including integrins $(11,12)$. It has been reported that the TSPAN superfamily affects the malignant properties of cancer cells, including their proliferation, apoptosis, metastasis, infiltration and cell-cell aggregation $(13,14)$. TSPAN1 has been identified as a member of the TSPAN family (15) and previous studies have revealed that TSPAN1 is highly expressed in gastric, colon, liver and esophageal cancers $(13,16,17)$. TSPAN1 has also been demonstrated to be important in gastric and colon cancer cell invasion and metastasis $(18,19)$. However, the role of TSPAN1 in PC, specifically in PC cell migration and invasion, is yet to be fully elucidated. 
In the present study, various methods including immunohistochemistry (IHC), reverse transcription-quantitative polymerase chain reaction (RT-qPCR) and western blotting were utilized to determine and assess the expression of TSPAN1 in human PC tissue samples, respective adjacent normal pancreatic tissue samples and in human pancreatic ductal adenocarcinoma (PDAC) cell lines. Furthermore, RT-qPCR and western blotting were performed to assess the expression of TSPAN1 following transfection with TSPAN1 small interfering RNAs (siRNAs) and pLNCX-TSPAN1-cDNA recombinant plasmids in human PC cell lines. Subsequently, cell migration and invasion were assessed via Transwell assays. The expression of matrix metalloproteinase (MMP2) and MMP9 were also determined and the molecular mechanism of TSPAN1 in human PC cell migration and invasion was further examined by employing phospholipase $C \gamma$ (PLC $\gamma$ ) siRNA.

\section{Materials and methods}

Tissues, cell lines and cell culture. The following PC cell lines SW1990, BxPC3, Capan1 and PANC-1, 293 and the immortalized non-tumorigenic human normal pancreatic epithelial cell line (HPDE) were purchased from Shanghai Institute of Biochemistry and Cell Biology, Chinese Academy of Sciences (Shanghai, China). Cells were grown in Dulbecco's modified Eagle's medium (DMEM; Invitrogen; Thermo Fisher Scientific, Inc., Waltham, MA, USA) supplemented with $10 \%$ fetal calf serum (FCS; Invitrogen; Thermo Fisher Scientific, Inc.) in a humidified incubator at $37^{\circ} \mathrm{C}$ under $5 \% \mathrm{CO}_{2}$. Cells were passaged until they reached $70-80 \%$ confluence using $0.25 \%(\mathrm{w} / \mathrm{v})$ trypsin solution in $0.02 \%(\mathrm{w} / \mathrm{v})$ EDTA. A total of 20 pairs of human PC tissue samples and their respective adjacent normal pancreatic tissue samples were obtained from China Medical University (Liaoning, China). Official written informed consent was obtained from each patient. A total of 95 patients were recruited between June 2015 and June 2016 (52 males and 43 females; age, 39-81 years). PC diagnosis was confirmed in all patients included in the present study. Patients had not undergone radiation therapy or chemotherapy. Tissue samples were collected during routine surgery performed in the Gastrointestinal Surgery Department of Cancer Hospital of China Medical University and the Department of General Surgery, Shenjing Hospital of China Medical University. The present study was approved by the Ethical Committee of China Medical University.

IHC. The expression of TSPAN1 in tissue samples was detected via IHC staining. Tissue was fixed with $10 \%$ formalin (Sigma-Aldrich; Merck KGaA, Darmstadt, Germany) at room temperature for $18 \mathrm{~h}$, paraffin-embedded using a tissue processor (Model, ATP700; Histo-Line laboratories, Milan, Italy) and sectioned on a microtome (Model, Leica RM 2165, Germany) to $4 \mu \mathrm{m}$ thick sections. Samples were collected on silane-coated slides for immunohistochemical staining. The tissue sections were sequentially deparaffinized and rehydrated. Antigens were then retrieved at $95^{\circ} \mathrm{C}$ using $10 \mathrm{mM}$ citrate buffer ( $\mathrm{pH}$ 6.0; Merck KGaA). Freshly prepared 3\% $\mathrm{H}_{2} \mathrm{O}_{2}$ was used to quench endogenous peroxidase activity. Blocking was subsequently performed with $10 \%$ normal serum
(Invitrogen; Thermo Fisher Scientific, Inc.) at room temperature for $1 \mathrm{~h}$. The sections were then sequentially incubated with anti-TSPAN1 primary antibodies (1:200; cat. no. NBP2-33867; Bio-Techne, Minneapolis, MN, USA) at $37^{\circ} \mathrm{C}$ for $2 \mathrm{~h}$. Tissues were then incubated with biotinylated goat anti-rabbit $\mathrm{IgG}$ secondary antibodies (1:1,000; cat. no. BAF008; R\&D Systems, Inc., Minneapolis, MN, USA) at room temperature for $30 \mathrm{~min}$ and washed with PBS. This was followed by incubation with horseradish peroxidase (HRP)-conjugated streptavidin (1:2,000; cat. no. N100; Thermo Fisher Scientific, Inc.) for $5 \mathrm{~min}$ at room temperature. Immunostaining was developed using the 3,3-Diaminobenzidine substrate (Gene Tech Co., Ltd., Hong Kong, China) for $10 \mathrm{~min}$ at room temperature. Sections were then counterstained with hematoxylin (Invitrogen; Thermo Fisher Scientific, Inc.) at room temperature for $3 \mathrm{~min}$. Sections that were incubated with PBS only served as the negative control. Cells were examined under an inverted-TS100 microscope (magnification, x200; Nikon Corporation, Tokyo, Japan) and the Aperio Scanscope XT system (Aperio, San Diego, CA, USA) was used to analyze the stained tissues sections.

$R T$-qPCR. An RNeasy Mini kit (Qiagen $\mathrm{GmbH}$, Hilden, Germany) was used to extract RNA from cells (HPDE, SW1990, BxPC3, Capan1, PANC-1, 293 cells, the transfected SW1990 and the transfected BxPC3 cells) following the manufacturer's protocol. Reverse transcription (RT) was performed as follows: A mixture of $2 \mu \mathrm{g}$ total RNA and $1 \mu \mathrm{l}$ Oligo (dT) was heated to $70^{\circ} \mathrm{C}$ for $5 \mathrm{~min}$, rapidly cooled on ice and mixed with a reaction mixture containing $5 \mu 1 \mathrm{M}-\mathrm{MLV}$ RT 5X Buffer (cat. no. M531A; Promega Corporation, Madison, WI, USA), $0.5 \mu \mathrm{l}$ deoxyribonucleotide triphosphate $(25 \mu \mathrm{M}$; cat. no. U1240; Promega Corporation), $0.7 \mu 1$ RNAse inhibitor ( $2500 \mathrm{U}$; RNasin ${ }^{\circledR}$; cat. no. N211A; Promega Corporation) and $1 \mu 1$ M-MLV reverse transcriptase (10,000 U; cat. no. M170A; Promega Corporation). The reaction volume was made up to $25 \mu 1$ with RNA-free water, and then incubated at $42^{\circ} \mathrm{C}$ for $1 \mathrm{~h}$ and then $70^{\circ} \mathrm{C}$ for $10 \mathrm{~min}$. Real-time PCR was performed using a total reaction volume of $20 \mu \mathrm{l}$, which included $2 \mathrm{X}$ Mix SYBR-Green I (10 $\mu \mathrm{l}$; Promega Corporation), primer $(0.25 \mu \mathrm{l}$, 10 pmol/l), template DNA $(1 \mu \mathrm{l})$ and sterile water. RT-qPCR was performed using the Applied Biosystems 7900HT thermal cycler instrument (Applied Biosystems; Thermo Fisher Scientific, Inc.). The following thermocycling conditions were used: Initial denaturation at $95^{\circ} \mathrm{C}$ for $2 \mathrm{~min}$, followed by 50 cycles of $95^{\circ} \mathrm{C}$ for $15 \mathrm{sec}$ and then $60^{\circ} \mathrm{C}$ for $45 \mathrm{sec}$. Target gene expression were assessed by calculating fold change using the formula: Fold change $=2-[\mathrm{Cq}$ (control) gene $\mathrm{X}-\mathrm{Cq}$ (control) actin] [Cq (activated) gene $\mathrm{X}-\mathrm{Cq}$ (activated) actin] (20). The following primer sequences were utilized: Human TSPAN1 forward, 5'-CGTTGTGGTCTTTGCTCTTG-3' and reverse, 5'-TTCTTGATGGCAGGCACTAC-3'; Human MMP2 forward, 5'-CTCGGTAGGGACATGCTAAGTAGAG-3' and reverse, 5'-CCTCTGGAGGTTCGACGTGA-3'; Human MMP9 forward 5'-TCATGAGGAAGAGCTCTGAGT-3' and reverse 5'-TCATGAGGAAGAGCTCTGAGT-3'; and Human GAPDH forward, 5'-GAAGGTGAAGGTCGGAGTC-3' and reverse, 5'-GAAGATGGTGATGGGATTTC-3'. The relative mRNA expression of TSPAN1, PLC $\gamma$ and MMPs were normalized to the respective endogenous GAPDH mRNA expression. 
Western blot analysis. Radioimmunoprecipitation assay buffer and a protease inhibitor were used to lyse cells and collect protein (Sigma-Aldrich; Merck KGaA) (21). A BCA assay (Thermo Fisher Scientific, Inc.) was used to quantify protein concentration. A total of $50 \mu \mathrm{g}$ of protein from each sample was denatured, resolved using 10\% SDS-PAGE gradient gel (EMD Millipore, Billerica, MA, USA) and electro-blotted to a polyvinylidene difluoride(PVDF) membrane (EMD Millipore). Membranes were then incubated with 5\% non-fat milk for $1 \mathrm{~h}$ at room temperature and stained with primary antibodies against TSPAN1 (1:300; cat. no. NBP2-33867; Bio-Techne) MMP2 (1:500; cat. no. 4022; Cell Signaling Technology, Inc., Danvers, MA, USA), PLC $\gamma 1$ (1:500; cat. no. 14008; Cell Signaling Technology, Inc.), phosphorylated PLC $\gamma 1$ (1:500; cat. no. 5690; Cell Signaling Technology, Inc.) and anti- $\beta$-actin $(1: 1,000$; cat. no. NB600-503; Novus Biologicals, LLC, Littleton, CO, USA) at $4^{\circ} \mathrm{C}$ overnight. The PVDF membrane was washed with TBST three times and incubated with HRP-conjugated secondary antibodies (HRP-linked anti-rabbit IgG antibodies; 1:3,000; cat. no. NB710-57836; Novus Biologicals, LLC) for $90 \mathrm{~min}$ at room temperature. Enhanced chemiluminescence (Pierce; Thermo Fisher Scientific, Inc.) was used to develop protein signals. A scanning densitometer along with Quantity One Software (version 4.4.1; Bio-Rad Laboratories, Inc., Hercules, CA, USA) was used to quantify the optical density of each protein band. Protein band density was normalized to the corresponding $\beta$-actin density.

Cytosolic protein was isolated from particulate-conjugated protein using the digitonin separation method from the transfected SW1990 and BxPC3 cells (22). Cells were collected and re-suspended in $1 \mathrm{ml}$ saline solution (1 mM EDTA, $150 \mathrm{mM} \mathrm{NaCl}, 1 \mathrm{mM}$ PMSF, $2 \mathrm{mM}$ EGTA, $1 \mu \mathrm{g} / \mathrm{ml}$ aprotinin, $10 \mu \mathrm{g} / \mathrm{ml}$ leupeptin, and $100 \mu \mathrm{g} / \mathrm{ml}$ digitonin) with occasional shaking for $10 \mathrm{~min}$. Cells were then centrifuged at $13,000 \mathrm{x} \mathrm{g}$ for $5 \mathrm{~min}$ at $4^{\circ} \mathrm{C}$ and the resulting supernatant contained the cytosolic proteins. The cell pellet was dissolved in $1 \mathrm{ml}$ lysis buffer (pH 7.4, 1 mM EDTA, 10 mM PBS, 1\% Triton X-100, $2 \mathrm{mM}$ EGTA, $1 \mathrm{mM}$ PMSF, $0.1 \%$ SDS, $1 \mu \mathrm{g} / \mathrm{ml}$ aprotinin and $10 \mu \mathrm{g} / \mathrm{ml}$ leupeptin) and the membrane protein was obtained (particulate-conjugated proteins). A total of $80 \mu \mathrm{g}$ of protein was separated via SDS-PAGE and transferred to a PVDF membrane as aforementioned. The expression of PLC $\gamma 1$ and phosphorylated PLC $\gamma 1$ were detected using western blotting as aforementioned.

Knockdown of TSPAN1 in SW1990 and BxPC3 cells by RNAi. The downregulation of TSPAN1 in SW1990 and BxPC3 cells was achieved using specific siRNAs targeting TSPAN1 (TSPAN1 siRNA; cat. no. 4392420; Thermo Fisher Scientific, Inc.). Cells transfected with scramble siRNA (cat. no. 4390846; Thermo Fisher Scientific, Inc.) were used as the control. The TSPAN1 siRNA group sequences were: 5'-GGCUCACGACCAAAAAGUAtt-3' (sense) and 5'-UAC UUUUUGGUCGUGAGCCtt-3' (antisense). The scramble siRNA group sequences were: 5'-UUCUCCGAACGUGUC ACGUdTdT-3' (sense), 5'-ACGUGACACGUUCGGAGAAdT dT-3' (antisense). The transfection of siRNAs into SW1990 and BxPC3 cells was performed following the manufacturer's protocol. SW1990 and BxPC3 cells were re-suspended at a density of $1 \times 10^{5}$ cells $/ \mathrm{ml}$ in DMEM medium. The RNAi MAX
Lipofectamine transfection agent (Ambion; Thermo Fisher Scientific, Inc.), siRNA (20 nM) and DMEM medium were mixed to prepare transfection complexes. SW1990 and BxPC3 cells were then mixed with the transfection complexes, seeded in 6-well $\left(2 \times 10^{5}\right.$ cells/well) plates (Nalge Nunc International, Rochester, NY, USA) and incubated for $24 \mathrm{~h}$ at $37^{\circ} \mathrm{C}$. RNA analysis was conducted on cells $48 \mathrm{~h}$ post-transfection, while protein analysis was performed $72 \mathrm{~h}$ post-transfection. The silencing efficiency of TSPAN1 siRNA was confirmed using RT-qPCR and western blotting. The transfection method of PLC $\gamma$ siRNA (cat. no. 1299001; Thermo Fisher Scientific, Inc.) into 293, SW1990 and BxPC3 cells, along with the determination of silencing efficacy were the same as aforementioned.

Overexpression of TSPAN1 in SW1990 and BxPC3 cells. A TSPAN1 cDNA clone plasmid was purchased from Beijing Zeping Bioscience \& Technology Co., Ltd. (Beijing, China; cat. no. HG 13073-UT). Recombinant plasmids (pLNCX-TSPAN1-cDNA) were constructed and sequenced by Bio Sune Biotechnologies (Shanghai, China). A total of $10 \mu \mathrm{g}$ of pLNCX-TSPAN1-cDNA recombinant plasmid was transfected into 293, SW1990 and BxPC3 cells via the calcium phosphate precipitation method (23). SW1990, 293 and BxPC3 cells were grown on a $35 \mathrm{~mm}$ cell culture dish until they reached $70-80 \%$ confluence. The DNA-calcium phosphate precipitation transfection complexes were prepared by mixing plasmid DNA and $2 \mathrm{M} \mathrm{CaCl}_{2}$ (Sigma-Aldrich; Merck KGaA) in HEPES buffered saline (included $50 \mathrm{mM}$ HEPES, $280 \mathrm{mM} \mathrm{NaCl}, 1.5 \mathrm{mM} \mathrm{Na} \mathrm{HPO}_{4}$; $\mathrm{pH}$ 7.1; Sigma-Aldrich; Merck KGaA). The transfection complexes were subsequently added into the cells drop by drop and incubated for $8 \mathrm{~h}$ at $37^{\circ} \mathrm{C}$. Fresh DMEM medium was then added for cell culture at $37^{\circ} \mathrm{C}$. The pLNCX plasmid was transfected into cells to serve as the control. At $48 \mathrm{~h}$ post-transfection, $0.25 \%$ (w/v) trypsin solution in $0.02 \%$ (w/v) EDTA was used to harvest cells, which were then cultured in DMEM medium with G418 $(500 \mathrm{mg} / \mathrm{l}$; Thermo Fisher Scientific, Inc.) to screen positive transfection clones. Following 2-3 weeks, positive clones were cultured in DMEM supplemented with $20 \%$ (v/v) heat-inactivated calf serum (Invitrogen; Thermo Fisher Scientific, Inc.) and G418 $(200 \mu \mathrm{g} / \mathrm{ml})$ at $37^{\circ} \mathrm{C}$ in $5 \% \mathrm{CO}_{2}$.

Assessment of cell migration and cell invasion in vitro by Transwell assay. Cell migration was measured using the BD Falcon HTS multi-well insert system (pore size, $8.0 \mu \mathrm{m}$, BD Biosciences, San Jose, CA, USA) (24). DMEM medium supplemented with $10 \%$ FCS at a volume of $500 \mu \mathrm{l}$ was added to the lower chamber of the wells. Transfected SW1990 and BxPC3 cells suspended in serum-free DMEM medium were added into the insert at a density of $1.5 \times 10^{5}$ cells $/ \mathrm{ml}$. Non-migrated cells (the cells remaining in the insert) were removed following incubation for $6 \mathrm{~h}$ at $37^{\circ} \mathrm{C}$ by gently scraping with cotton swabs. Cells at the bottom of the insert, which represent the migrated cells, were fixed with $4 \%$ formaldehyde for $20 \mathrm{~min}$ at room temperature. Fixed cells were then stained with $0.1 \%$ crystal violet for $30 \mathrm{~min}$ at room temperature. Excess stain was removed by washing the inserts with Milli-Q water (Merck KGaA). Filters were then dried overnight. The number of migrated cells was estimated by counting cells in five random fields and images were obtained using light 

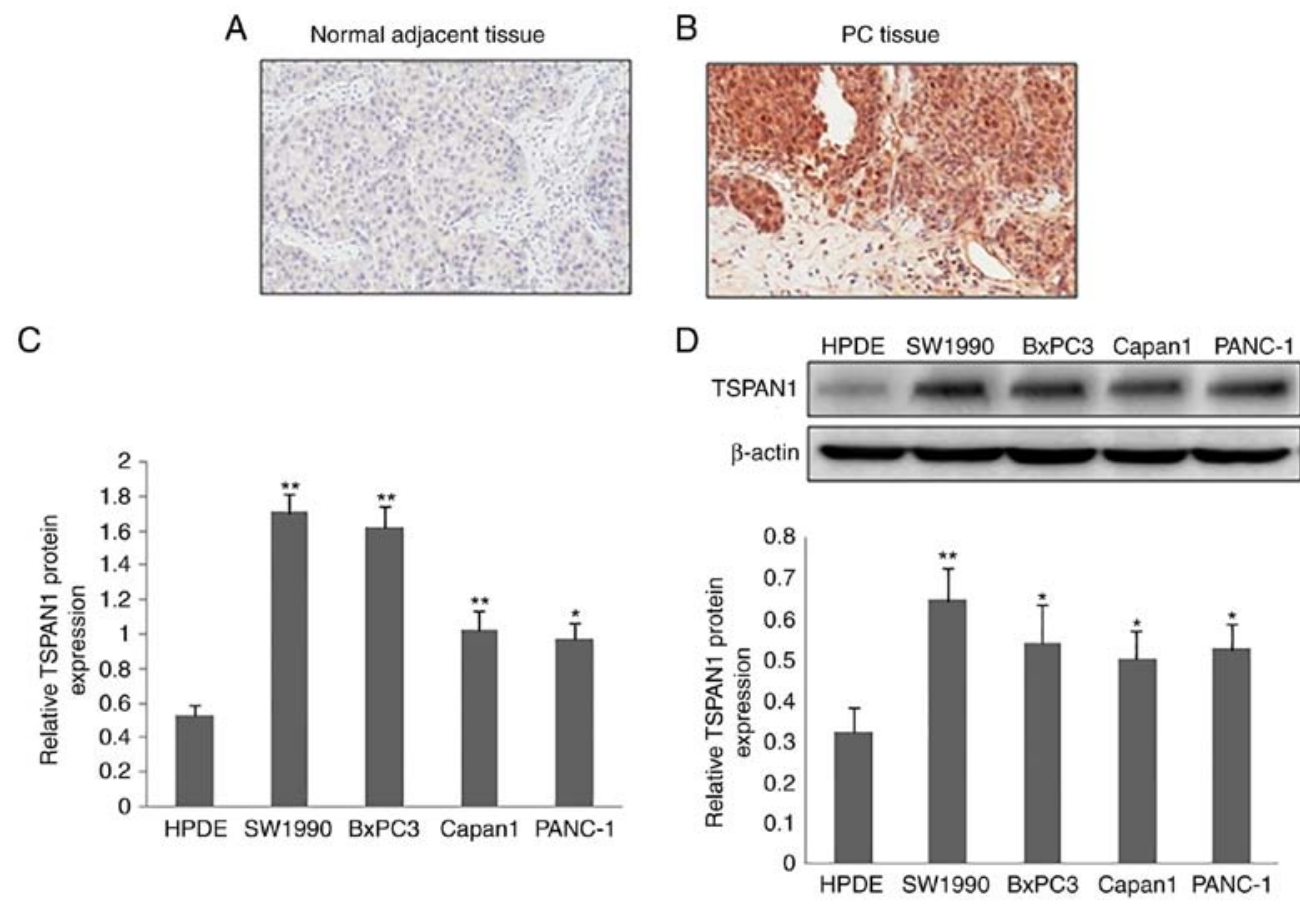

Figure 1. Expression of TSPAN1 in human pancreatic tumor tissue samples and cell lines. The expression and location of TSPAN1 in human pancreatic tumor tissue samples and adjacent normal pancreatic tissue samples were detected using the immunohistochemistry method (magnification, x200). (A) TSPAN1 expression in adjacent non-cancer tissues. (B) High expression of TSPAN1 in pancreatic cancer tissue samples. (C) The mRNA expression of TSPAN1 was assessed via reverse transcription polymerase chain reaction in SW1990, BxPC3, Capan1 and PANC-1 cell lines, and the normal pancreatic cell line HPDE. (D) The protein expression of TSPAN1 in the indicated cell lines was analyzed using western blotting (upper panel) and quantified (lower panel). Data are expressed as the mean \pm standard deviation $(n=3)$. ${ }^{*} \mathrm{P}<0.05$ and $^{* *} \mathrm{P}<0.01$ vs. HPDE. TSPAN1, Tetraspanin 1.

microscopy and captured using a camera (magnification, $\mathrm{x} 100$; Nikon Digital Camera Dxm 1200F; Nikon Corporation).

The cell invasion assay was performed using BioCoat Matrigel invasion chambers (BD Biosciences) (25). A total of $50 \mu \mathrm{g}$ of reduced serum Matrigel (BD Biosciences) was added to the upper chamber of the inserts to coat the Transwell membrane. Transfected SW1990 and BxPC3 cellsin serum-free DMEM $\left(1 \times 10^{6}\right)$ were added into the upper chambers. DMEM supplemented with $10 \%$ FCS was added to the lower chamber and served as a chemoattractant. Following $48 \mathrm{~h}$ of incubation at $37^{\circ} \mathrm{C}$, the membrane facing the lower chamber (where invaded cells were present) was gently removed and mounted on a glass slide. The subsequent steps for fixation, staining and measuring the level of cell invasion were as aforementioned for the migration assay.

Statistical analysis. The results were expressed as the mean \pm standard deviation of at least three independent experiments performed in triplicate. SPSS 17.0 software (SPSS, Inc., Chicago, IL, USA) was used to determine significant differences. For comparisons between two groups, a two-tailed Student's t-test was performed. One-way analysis of variance followed by a Tukey's post hoc test was used to assess significant differences among multiple groups. $\mathrm{P}<0.05$ was considered to indicate a statistically significant difference.

\section{Results}

Expression of TSPAN1 in clinical PC tissue samples and cell lines. The protein expression of TSPAN1 in clinical tissue samples of PC was detected via IHC staining. Compared with normal adjacent tissue samples, a markedly increased TSPAN1expression was observed in the PC tissue samples (Fig. 1A and B). TSPAN1 expression was predominantly observed in the cytoplasm of the cells. The mRNA expression of TSPAN1, as determined by RT-qPCR, was significantly increased in PC cell lines (SW1990, BxPC3, Capan1 and PANC-1) compared with the normal pancreatic cell line (HPDE; Fig. 1C). Similarly, TSPAN1 protein expression in the PC cell lines (SW1990, BxPC3, Capan1 and PANC-1) was higher when compared with the normal pancreatic cell line (HPDE; Fig. 1D).

Knockdown of TSPAN1 expression via TSPAN1 siRNA transfection. TSPAN1-siRNAs were transfected into human PC cell lines (SW1990 and BxPC3). The silencing efficiency of TSPAN1-siRNAs at the gene and protein level was confirmed by RT-qPCR and western blotting, respectively. TSPAN1 mRNA was significantly decreased in TSPAN1-siRNA transfected cells when compared with the control cells (those transfected with scrambled siRNA; Fig. 2A). Consistently, a significant downregulation of TSPAN1 protein was observedinTSPAN1-siRNA-transfected cells compared with the control cells (Fig. 2B).

Overexpression of TSPAN1 in SW1990 and BxPC3 cells. The expression of TSPAN1 in PC cell lines (SW1990 and BxPC3) transfected with pLNCX-TSPAN1-cDNA recombinant plasmid and pLNCX plasmid was determined via RT-qPCR and western blotting (Fig. 2C and D). Cells transfected with 
A
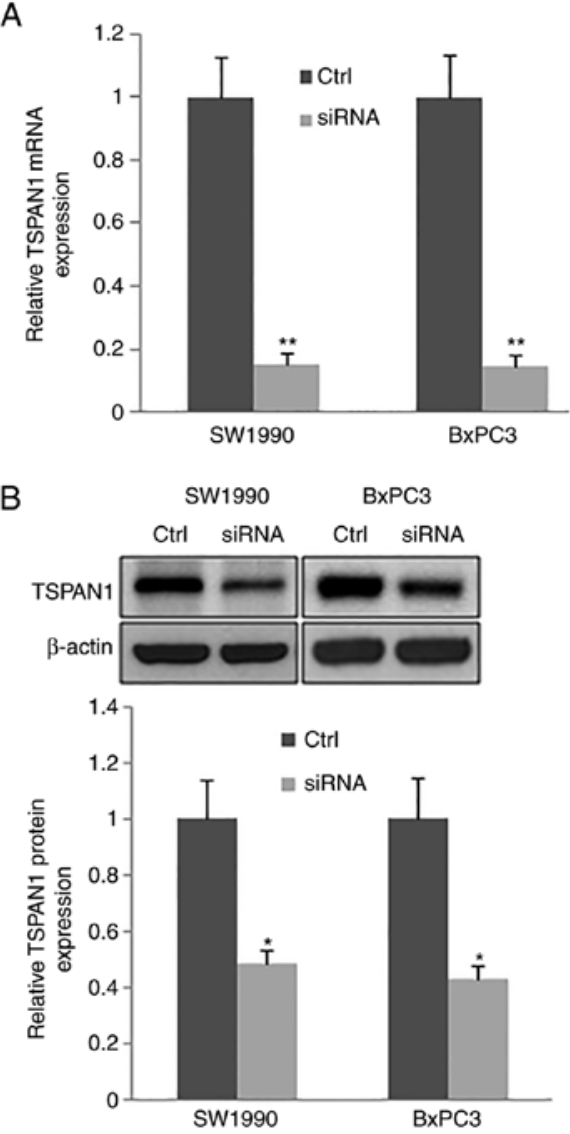

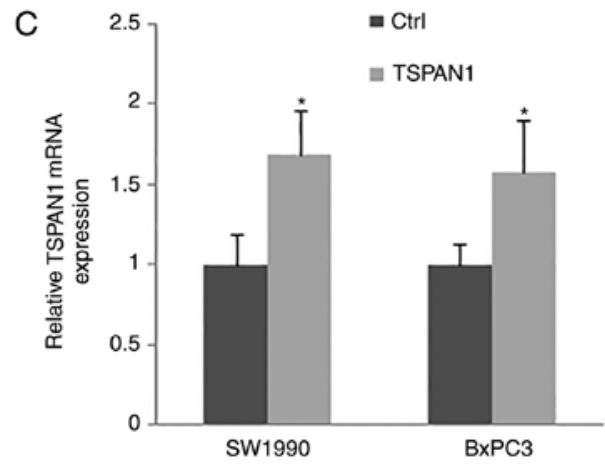

D
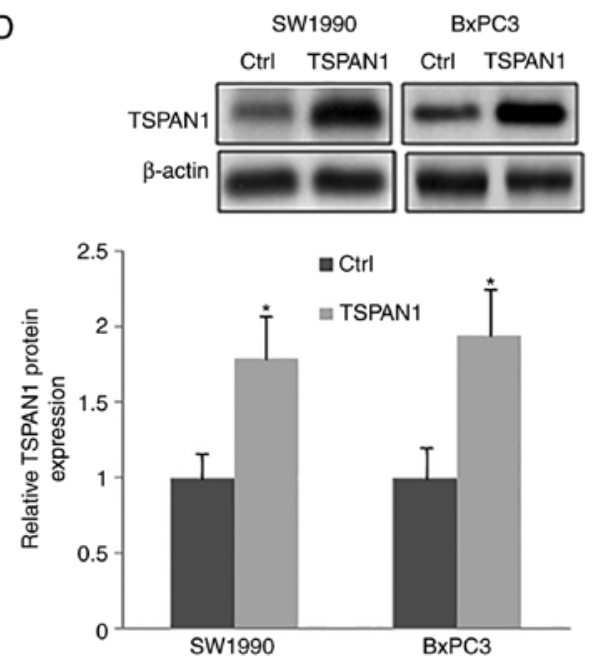

Figure 2. Successful transfection of siTSPAN1 and pLNCX-TSPAN1-cDNA plasmid into SW1990 and BxPC3 cell lines and analysis of the transfection effects. (A) siRNA targeted to TSPAN1 was transfected into SW1990 and BxPC3 cells (siRNA group); Scrambled siRNA-transfected cells were used as control (Ctrl). RT-qPCR was performed to analyze the mRNA levels of TSPAN1. (B) Western blot analysis was performed to detect the protein level of TSPAN1 following TSPAN1-siRNAs transfection (upper panel) and was subsequently quantified (lower panel). (C) The pLNCX-TSPAN1-cDNA recombinant plasmid was transfected into SW1990 and BxPC3 cells (TSPAN1 group), and plasmid pLNCX was transfected into SW1990 and BxPC3 cells as the control group (Ctrl). The mRNA levels of TSPAN1 were analyzed via RT-qPCR. (D) The protein levels of TSPAN1 were detected using western blottingfollowingpLNCX-TSPAN1-cDNA transfection (upper panel) and then quantified (lower panel). ${ }^{*} \mathrm{P}<0.05$ and ${ }^{* *} \mathrm{P}<0.01$ vs. Ctrl (n=3). TSPAN1, Tetraspanin 1; RT-qPCR, reverse transcription-quantitative polymerase chain reaction; siRNA, small interfering RNA; Ctrl, control.

pLNCX were used as the control. The mRNA and protein expression of TSPAN1 was significantly upregulated in cells transfected with pLNCX-TSPAN1-cDNA compared with pLNCX-transfected cells. The results indicate that the TSPAN1-cDNA recombinant plasmid was successfully transfected into SW1990 and BxPC3 cells.

TSPAN1 inhibits SW1990 and BxPC3 cell migration and invasion, and MMP2/9 expression. Cell migration and invasion were determined in PC cell lines (SW1990 and BxPC3). Following TSPAN1-siRNA transfection, the downregulation of TSPAN1 significantly decreased cell migration and invasion compared with the control cells transfected with scrambled siRNA (Fig. 3A and B). Consistent with this, TSPAN1 ectopic overexpression in SW1990 and BxPC3 cells markedly increased cell migration and invasion in these cell lines (Fig. 3C and D). The results indicate that TSPAN1 knockdown suppresses PC cell migration and invasion.

Additionally, the expression of MMP2 at the mRNA and protein levels, as assessed via RT-qPCR and western blotting, respectively, were significantly decreased in TSPAN1 siRNA-transfected SW1990 and BxPC3 cells in comparison with scrambled siRNA transfected cells (Control). TSPAN1 ectopic overexpression in the same cell lines increased the expression of MMP2 at the mRNA and protein level (Fig. 4Ba and b). However, the mRNA levels of MMP9 were not changed following transfection with TSPAN1 siRNA or pLNCX-TSPAN1-cDNA in SW1990 and BxPC3 cells (Fig. 4Ac and $\mathrm{Bc}$ ). These results indicate that TSPAN1 knockdown suppresses the expression of MMP2, but not that of MMP9.

Overexpression of TSPAN1 in PC cell lines promotes cell migration, invasion and MMP2 expression via PLC $\gamma$. The present study assessed the effects of PLC $\gamma$ siRNA on PC cell migration and invasion and MMP2 expression following LNCX-TSPAN1-cDNA transfection. The expression of PLC $\gamma$ was significantly downregulated after PLC $\gamma$ siRNA was transfected into 293 cells, as determined by RT-qPCR and western blot analysis (Fig. 4C). Furthermore, the upregulated MMP2 expression, induced by the overexpression of TSPAN1 in the SW1990 and BxPC3 PC cell lines, was significantly reduced following the transfection of PLC $\gamma$ siRNA (Fig. 4D and E). Thus, the results indicate that the overexpression of TSPAN1 promotes the expression of MMP2 and may be dependent on PLC $\gamma$. In addition, the increased cell migration and invasion 
A

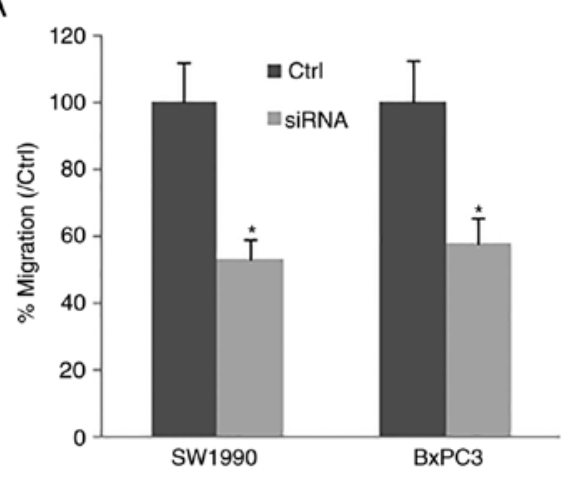

B

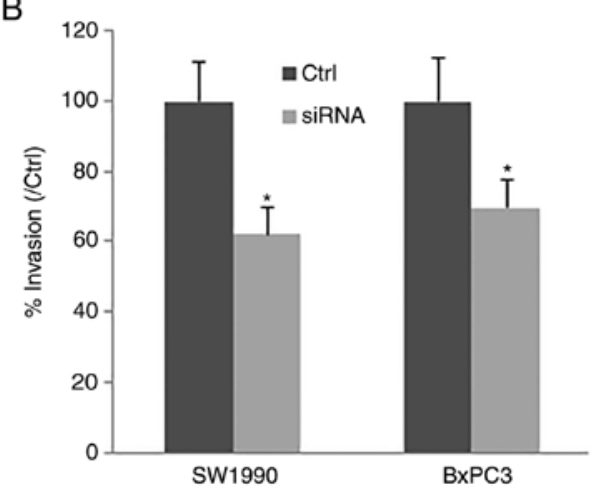

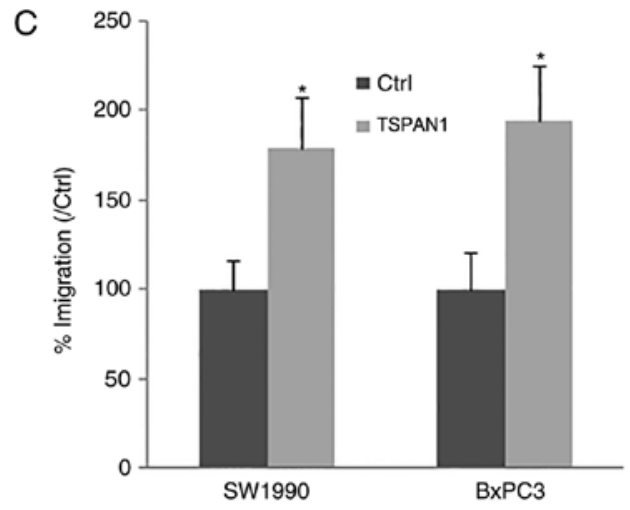

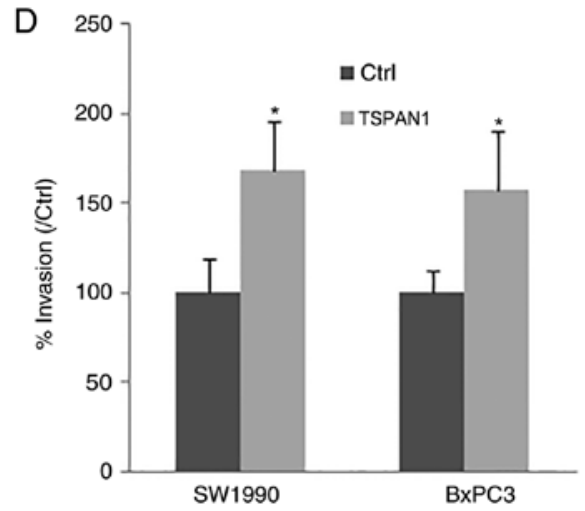

Figure 3. TSPAN1 modulates the migration and invasion of SW1990 and BxPC3 cells in vitro. cell migration and invasion was assessed using a Transwell asssay. In the presence of siTSPAN1 (siRNA group), the number of (A) migrating and (B) invading SW1990 and BxPC3 cells was reduced when compared with the control cells (scrambled siRNA-transfected cells; Ctrl group). Following pLNCX-TSPAN1-cDNA recombinant plasmid transfection into SW1990 and BxPC3 cells (TSPAN1 group), cell (C) migration and (D) invasion were analyzed; the plasmid pLNCX was transfected into SW1990 and BxPC3 cells to generate control cells (Ctrl). ${ }^{*} \mathrm{P}<0.05$ vs. $\mathrm{Ctrl}(\mathrm{n}=3)$. TSPAN1, Tetraspanin 1; siRNA, small interfering RNA; Ctrl, control.

induced by TSPAN1 overexpression was significantly attenuated following transfection with PLC $\gamma$ siRNA (Fig. 5A and B). Therefore, the increased cell migration and invasion of TSPAN1-overexpressing cells and the upregulation of MMP2 expression may be dependent on PLC $\gamma$.

To confirm this hypothesis, the effect of TSPAN1 siRNA transfection on the activation of PLC $\gamma$ was assessed. The proteins in the cytoplasm were isolated from particulate-conjugated proteins. The content of PLC $\gamma 1$ and phosphorylated PLC $\gamma 1$ were detected via western blotting. The results presented in Fig. 5C and D revealed that TSPAN1 siRNA transfection decreases the content of PLC $\gamma 1$ in the cytoplasm and decreases the content of PLC $\gamma 1$ and phosphorylated PLC $\gamma 1$ on the plasma membrane.

The results demonstrated that TSPAN1 siRNA transfection inhibits the translocation and phosphorylation of PLC $\gamma 1$, and thus inhibits the activation of PLC $\gamma 1$. Thus, the aforementioned results indicate that TSPAN1 may affect PC cell migration and invasion as well as MMP2 expression via the activation of PLC $\gamma$.

\section{Discussion}

$\mathrm{PC}$ is the most common malignancy of the pancreas (1-3). PC arising from the ductal epithelium of the pancreas, otherwise known as PDAC, is the most frequently diagnosed type of PC, accounting for $\sim 80-90 \%$ of all cases (1-3). PC exhibits high rates of metastasis and mortality. As such, the 5-year survival rate following PC diagnosis is poor, often at $<6 \%(1,2)$. Therefore, effective therapeutic strategies are urgently required for PC.

TSPANs are a membrane protein superfamily that has four transmembrane domains (9). This four-transmembrane structure constructs intracellular-membrane-extracellular signaling pathways and facilitates the binding and interactions of cell surface signaling molecules, including integrin and cadherin (10-12). It has been demonstrated that the TSPAN superfamily is implicated in essential cellular activities, including cell survival, growth, proliferation, differentiation, migration, signal transduction and motility (9-14). TSPAN1 is a novel member of the TSPANs family (15). Previous studies have reported an overexpression of TSPAN1 in various types of cancers, including liver, colon and gastric cancer (16-19). In the present study, the results indicated that TSPAN1 was highly expressed in human PC tissue samples compared with adjacent normal pancreatic tissue samples. This observation was consistent in cell lines, where the expression of TSPAN1 was significantly higher in human PC cells (SW1990, BxPC3, Capan1 and PANC-1) when compared with that observed in HPDE, a normal pancreatic cell line.

To date, the function of TSPAN1 in PC is still undetermined. Previous reports have revealed that TSPAN1is positively correlated with clinical stage and negatively correlated with the survival rate of patients with cancer $(25,26)$. In colon cancer and skin carcinoma cells, TSPAN1 has been revealed to inhibit cell survival, proliferation, migration and invasion $(19,27)$. Furthermore, TSPAN1 has been demonstrated to promote cell 
A

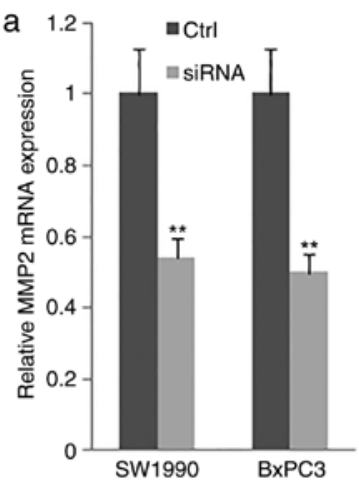

B a

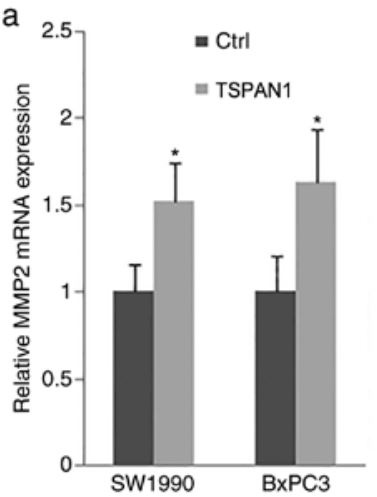

C
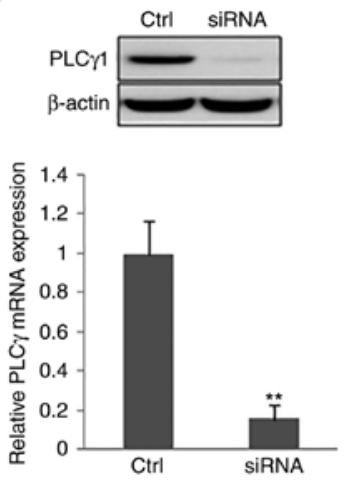

b

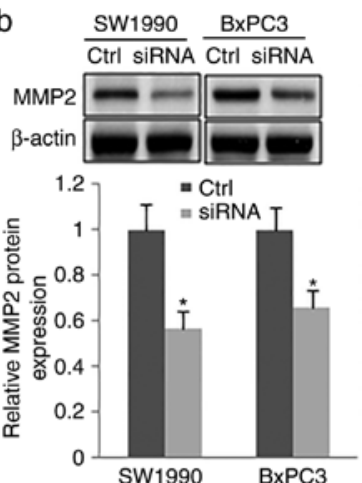

b
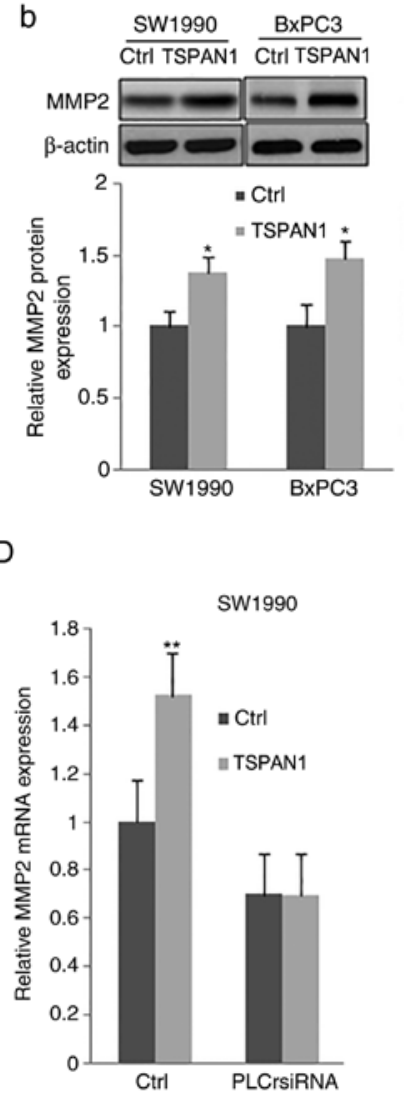

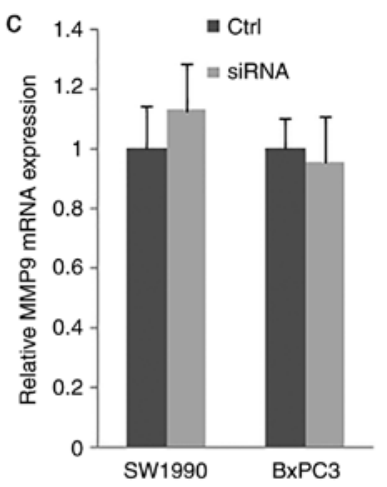

C

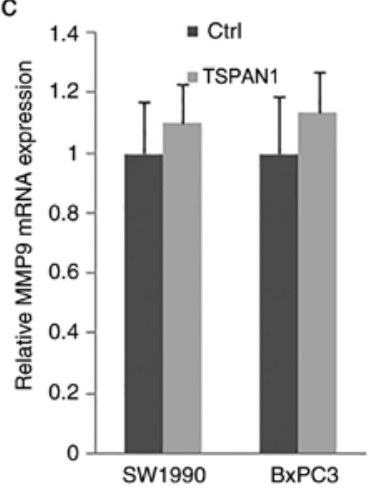

CtrI PLCrsiRnA

Ctrl TSPAN1 CtrI TSPAN1
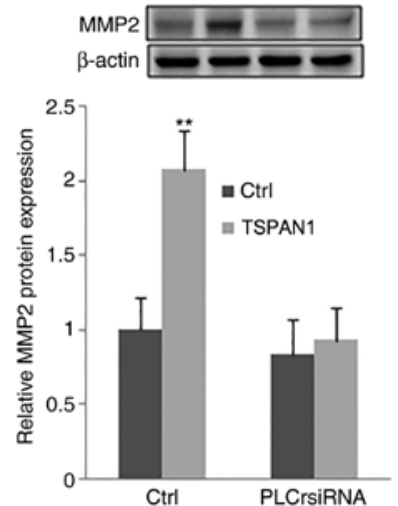

E

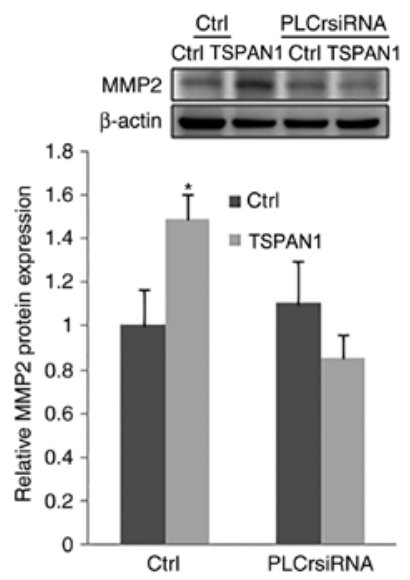

Figure 4. TSPAN1 modulates MMP2 expression via PLC $\gamma$. SW1990 and BxPC3 cells were transfected with siRNAs targeted to TSPAN1 (siRNA group) and pLNCX-TSPAN1-cDNA recombinant plasmid (TSPAN1 group). (A) RT-qPCR and western blotting were performed to assess the expression of (a and b) MMP2 and (c) MMP9 at the mRNA and protein level following TSPAN1 silencing in SW1990 and BxPC3 cells. Scrambled siRNA-transfected cells were used as controls (Ctrl). (B) The mRNA and protein expression of (a and b) MMP2 and (c) MMP9 was assessed following TSPAN1 overexpression in the indicated cells. Plasmid pLNCX transfected into SW1990 and BxPC3 cells was used as the control (Ctrl). (C) NIH3T3 cells were transfected with PLC $\gamma$ siRNA. RT-qPCR and western blotting was performed to detect the expression of PLC $\gamma$. Following PLC $\gamma$ siRNA transfection, the expression of MMP2 was detected in TSPAN1 overexpressed (D) SW1990 and (E) BxPC3 cells. " $\mathrm{P}<0.05$ and ${ }^{* *} \mathrm{P}<0.01$ vs. Ctrl. TSPAN1, Tetraspanin 1; RT-qPCR, reverse transcription-quantitative polymerase chain reaction; siRNA, small interfering RNA; Ctrl, control; MMP, matrix metalloproteinase; PLC $\gamma$, phospholipase C $\gamma$. 

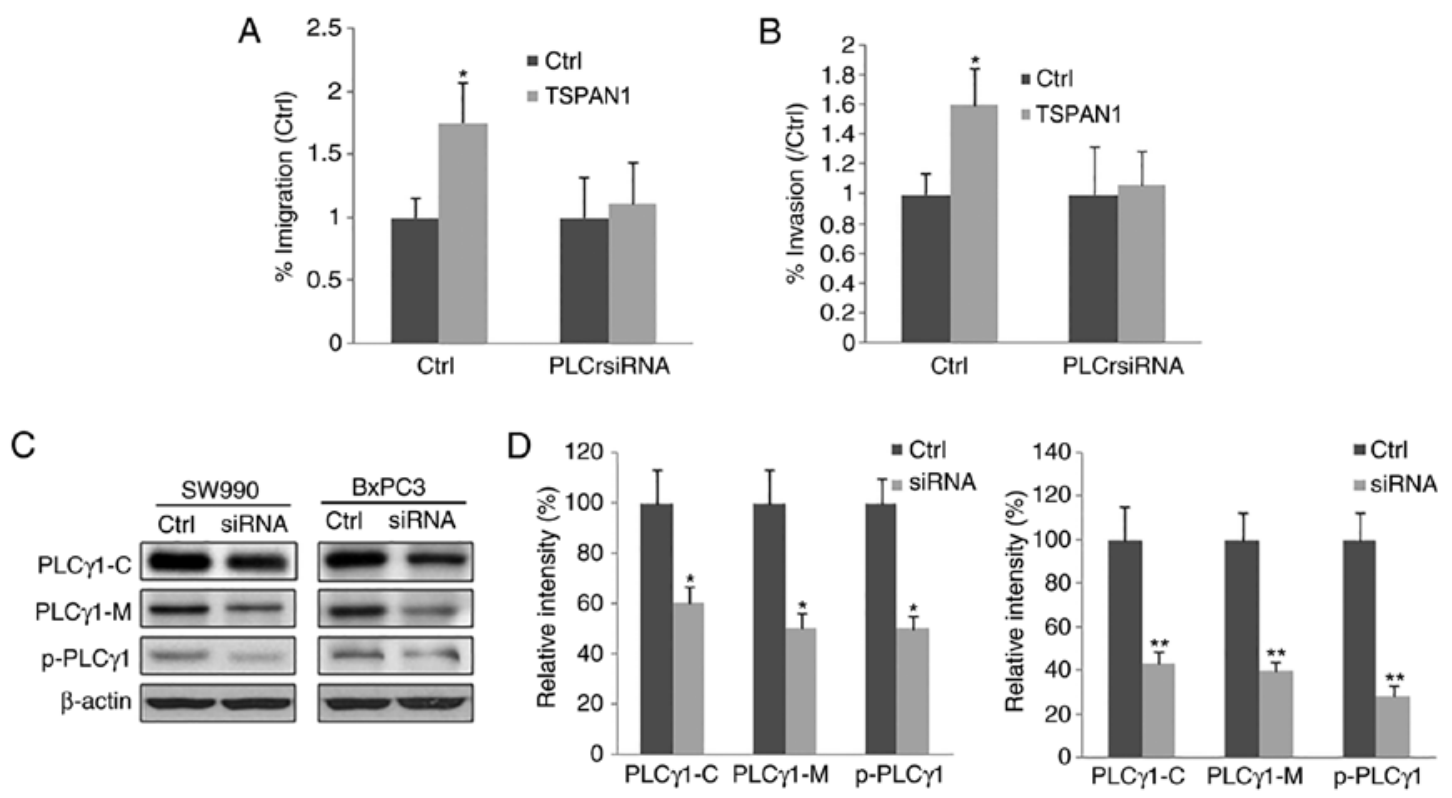

Figure 5. TSPAN1 upregulates the migration and invasion of PC cells via PLC $\gamma$. SW1990 and BxPC3 cells were transfected with pLNCX-TSPAN1-cDNA recombinant plasmid (TSPAN1 group). Plasmid pLNCX transfected into SW1990 and BxPC3 cells was used as the control (Ctrl). Following PLC $\gamma$ siRNA transfection, cell migration and invasion were assessed in SW1990 and BxPC3 cells overexpressing TSPAN1. The quantitative analysis of (A) cell migration and (B) cell invasion. Cells were permeabilized and centrifuged to isolate the cytosolic proteins and particulate-conjugated proteins. (C) Western blotting was then performed to assess their expression. (D) Analysis of the relative protein levels. ${ }^{*} \mathrm{P}<0.05$ and ${ }^{* *} \mathrm{P}<0.01$ vs. Ctrl ( $\mathrm{n}=3$ ). TSPAN1, Tetraspanin 1 ; siRNA, small interfering RNA; Ctrl, control; PLC $\gamma$, phospholipase C $\gamma$; PLC $\gamma 1$-C, PLC $\gamma 1$ in cytoplasm; PLC $\gamma 1-M$, PLC $\gamma 1$ conjugated with plasma membrane; p-PLC $\gamma 1$, phosphorylated PLC $\gamma 1$ conjugated with plasma membrane.

migration and invasion in human cervical tumor cells (28). The present study assessed the role of TSPAN1 in the progression of PC. The results demonstrated that the ectopic expression of TSPAN1 promoted PC cell migration and invasion. Consistent with the results of previous studies, the silencing of TSPAN1 suppressed PC cell migration and invasion $(12,27,29)$. These results indicate that TSPAN1 may be an oncogenic gene.

A complex, continuous multi-step process is involved in the invasion and metastasis of PC cells. Among these, the proteolytic degradation of the extracellular matrix (ECM) is an essential event (30). Membrane-type metalloproteinase directly or indirectly degrades various components of the ECM including collagens, fibronectin, laminins, HS proteoglycans and vitronectin $(31,32)$. MMP2 and MMP9, which are members of the MMP protein family, cleave collagen and other types of ECM $(33,34)$. Previous studies have revealed a close association between MMP2 and MMP9, and PC invasion and metastasis, which is further supported by the high expression of MMP2 and MMP9 demonstrated in PC tissue samples of the present study $(35,36)$. The present results also revealed that the knockdown of TSPAN1 significantly downregulated the expression of MMP2, but did not affect MMP9 mRNA expression. In addition, MMP2 expression in PC cells was markedly increased following TSPAN1 ectopic overexpression. Therefore, the downregulation of MMP2 expression maybe one way in which the knockdown of TSPAN1 inhibits PC cell migration and invasion. Furthermore, the present results indicated that PLC $\gamma$ siRNA significantly reduced cell migration and invasion, and the expression of MMP2 induced by TSPAN1 overexpression, indicating that TSPAN1 siRNA inhibits PC cell migration and invasion and that MMP2 expression may be dependent on PLC $\gamma$. To confirm this hypothesis, the effect of TSPAN1 siRNA transfection on the activation of PLC $\gamma$ was assessed. The results demonstrated that TSPAN1 siRNA transfection inhibited the translocation and phosphorylation of PLC $\gamma 1$, and thus inhibited the activation of PLC $\gamma 1$. In conclusion, the results of the present study indicate that the knockdown of TSPAN1 suppresses PC cell migration and cell invasion by downregulating MMP2 induced by PLC $\gamma$. The results indicate that TSPAN1 may serve as an important biomarker for the diagnosis and prognosis of PC. Therefore, TSPAN1-targeted siRNA may be a promising therapy for PC and other types of cancer with increased TSPAN1 expression. However, future studies should assess the feasibility of this therapy in animal models to confirm these results.

\section{Acknowledgements}

Not applicable.

\section{Funding}

The present study was funded by the Liaoning Doctoral Scientific Research Initial Foundation (grant no. 201601417), the Project of Shenyang Municipal Science and Technology Bureau (grant no. 18-014-4-75), the National Natural Science Foundation (grant no. 81672427) and the National Natural Science Foundation of China (grant no. 30973501).

\section{Availability of data and materials}

The datasets used and/or analyzed during the present study are available from the corresponding author on reasonable request. 


\section{Author's contributions}

XZ, GS and XT conceived and designed the present study. XZ, GS, FG and PL performed the experiments. FG, PL and HW analyzed the data and wrote the manuscript. GS and XT wrote the manuscript. All authors read and approved the final manuscript.

\section{Ethics approval and consent to participate}

The present study was performed after obtaining approval from China Medical University. Samples were taken from China Medical University with official written ethical consent from the patients.

\section{Patient consent for publication}

All patients provided their written informed consent for Publication and agreed to the publication of their associated data and any accompanying images as appropriate.

\section{Competing interests}

The authors declare that they have no competing interests.

\section{References}

1. Vincent A, Herman J, Schuick R, Hruban RH and Goggins M: Pancreatic cancer. Lancet 378: 607-620, 2011.

2. Siegel R, Ma J, Zou Z and Jemal A: Cancer statistics, 2014. CA Cancer J Clin 64: 9-29, 2014

3. Bosetti C, Bertuccio P, Negri E, La Vecchia C, Zeegers MP and Boffetta P: Pancreatic cancer: Overview of descriptive epidemiology. Mol Carcinog 51: 3-13, 2012.

4. Loos M, Kleeff J, Friess H and Büchler MW: Surgical treatment of pancreatic cancer. Ann N Y Acad Sci 1138: 169-180, 2008.

5. Mitchem JB, Hamilton N, Gao F, Hawkins WG, Linehan DC and Strasberg SM: Long-term results of resection of adenocarcinoma of the body and tail of the pancreas using radical antegrade modular pancreatosplenectomy procedure. J Am Coll Surg 214 $46-52,2012$.

6. Nakai Y, Isayama H, Sasaki T, Sasahira N, Tsujino T, Toda N, Kogure $\mathrm{H}$, Matsubara S, Ito Y, Togawa $\mathrm{O}$, et al: A multicentre randomised phase II trial of gemcitabine alone vs gemcitabine and S-1 combination therapy in advanced pancreatic cancer: GEMSAP study. Br J Cancer 106: 1934-1939, 2012.

7. Long Y, Sun Q, Wu J, Wang Y and Jiao S: Allogeneic cell-based immunotherapy combined with chemotherapy and targeted therapy in advanced pancreatic cancer with metastases: A case report. Oncol Lett 7: 1594-1598, 2014.

8. Hall WA, Colbert LE, Nickleach D, Switchenko J, Liu Y, Gillespie T, Lipscomb J, Hardy C, Kooby DA, Prabhu RS, et al: The influence of radiation therapy dose escalation on overall survival in unresectable pancreatic adenocarcinoma. J Gastrointest Oncol 5: 77-85, 2014.

9. Hemler ME: Tetraspanin functions and associated microdomains. Nat Rev Mol Cell Biol 6: 801-811, 2005.

10. Yáñez-Mó M, Barreiro O, Gordon-Alonso M, Sala-Valdés M and Sánchez-Madrid F: Tetraspanin-enriched microdomains: A functional unit in cell plasma membranes. Trends Cell Biol 19: 434-446, 2009.

11. Stipp CS, Kolesnikova TV and Hemler ME: Functional domains in tetraspanins proteins. Trends Biochem Sci 28: 106-122, 2003.

12. Liu L, He B, Liu WM, Zhou D, Cox JV and Zhang XA: Tetraspanin CD151 promotes cell migration by regulating integrin trafficking. J Biol Chem 282: 31631-31642, 2007.

13. Richardson MM, Jennings LK and Zhang XA: Tetraspanins and tumor progression. Clin Exp Metastasis 28: 261-270, 2011.

14. Pedro AL: Functional implications of tetraspanin proteins in cancer biology. Cancer Science 98: 1666-1677, 2007.

15. Garcia-España A, Chung PJ, Sarkar IN, Stiner E, Sun TT and Desalle R: Appearance of new tetraspanin genes during vertebrate evolution. Genomics 91: 326-334, 2008.
16. Guo Q, Xia B, Zhang F, Richardson MM, Li M, Zhang JS, Chen $\mathrm{F}$ and Zhang XA: Tetraspanin CO-029 inhibits colorectal cancer cell movement by deregulating cell-matrix and cell-cell adhesions. PLoS One 7: e38464, 2012.

17. Ke AW, Shi GM, Zhou J, Wu FZ, Ding ZB, Hu MY, Xu Y, Song ZJ, Wang ZJ, Wu JC, et al: Role of overexpression of CD151 and/or c-Met in predicting prognosis of hepatocellular carcinoma. Hepatology 49: 491-503, 2009.

18. Duan H, Qu L and Shou C: Activation of EGFR-PI3K-AKT signaling is required for Mycoplasma hyorhinis-promoted gastric cancer cell migration. Cancer Cell Int 14: 135, 2014.

19. Chen L, Yuan D, Zhao R, Li H and Zhu J: Suppression of TSPAN1 by RNA interference inhibits proliferation and invasion of colon cancer cells in vitro. Tumori 96: 744-750, 2010.

20. Livak KJ and Schmittgen TD: Analysis of relative gene expression data using real-time quantitative PCR and the $2^{-\Delta \Delta C_{\mathrm{T}}}$ method. Methods 25: 402-408, 2001.

21. Albrethsen J, Bøgebo R, Gammeltoft S, Olsen J, Winther B and Raskov $\mathrm{H}$ : Upregulated expression of human neutrophil peptides 1, 2 and 3 (HNP 1-3) in colon cancer serum and tumours: A biomarker study. BMC Cancer 5: 8, 2005.

22. Cordeiro $\mathrm{C}$ and Freire AP: Digitonin permeabilization of Saccharomyces cerevisiae cells for in situ enzyme assay. Anal Biochem 229: 145-148, 1995.

23. Chen CA and Okayama H: Calcium phosphate-mediated gene transfer: A highly efficient transfection system for stably transforming cells with plasmid DNA. Biotechniques 6: 632-638, 1988.

24. Takahashi M, Sugiura T, Abe M, Ishii K and Shirasuna K: Regulation of c-Met signaling by the tetraspanin KAI-1/CD82 affects cancer cell migration. Int J Cancer 121: 1919-1929, 2007.

25. Fei Y, Wang J, Liu W, Zuo H, Qin J, Wang D, Zeng H and Liu Z: CD151 promotes cancer cell metastasis via integrins $\alpha 3 \beta 1$ and a6ß1 in vitro. Mol Med Rep 6: 1226-1230, 2012.

26. Haeuw JF, Goetsch L, Bailly C and Corvaia N: Tetraspanin CD151 as a target for antibody-based cancer immunotherapy. Biochem Soc Trans 39: 553-558, 2011.

27. Chen L, Zhu Y, Li H, Wang GL, Wu YY, Lu YX, Qin J, Tuo J, Wang JL and Zhu J: Knockdown of TSPAN1 by RNA silencing and antisense technique inhibits proliferation and infiltration of human skin squamous carcinoma cells. Tumori 96: 289-295, 2010.

28. Hölters S, Anacker J, Jansen L, Beer-Grondke K, Dürst M and Rubio I: Tetraspanin 1 promotes invasiveness of cervical cancer cells. Int J Oncol 43: 503-512, 2013.

29. Lu Z, Luo T, Nie M, Pang T, Zhang X, Shen X, Ma L, Bi J, Wei G, Fang G and Xue X: TSPAN1 functions as an oncogene in gastric cancer and is downregulated by miR-573. FEBS Lett 589: 1988-1994, 2015.

30. Ellenrieder V, Adler G and Gress TM: Invasion and metastasis in pancreatic cancer. Ann Oncol 10 (Suppl 4): S46-S50, 1999.

31. Nagase H and Woessner JF Jr: Matrix metalloproteinases. J Biol Chem 274: 21491-21494, 1999.

32. Curran S and Murray GI: Matrix metalloproteinases in tumour invasion and metastasis. J Pathol 189: 300-308, 1999.

33. Bramhall SR, Neoptolemos JP, Stamp GW and Lemoine NR: Imbalance of expression of matrix metalloproteinases (MMPs) and tissue inhibitors of the matrix metalloproteinases (TIMPs) in human pancreatic carcinoma. J Pathol 182: 347-355, 1997.

34. Khokha R and Denhardt DT: Matrix metalloproteinases and tissue inhibitor of metalloproteinases: A review of their role in tumorigenesis and tissue invasion. Invasion Metastasis 9: 391-405, 1989.

35. Koshiba T, Hosotani R, Wada M, Fujimoto K, Lee JU, Doi R, Arii $S$ and Imamura M: Detection of matrix metalloproteinase activity in human pancreatic cancer. Surg Today 27: 302-304, 1997.

36. Nagakawa Y, Aoki T, Kasuya K, Tsuchida A and Koyanagi Y: Histologic features of venous invasion, expression of vascular endothelial growth factor and matrix metalloproteinase- 2 and matrix metalloproteinase-9, and the relation with liver metastasis in pancreatic cancer. Pancreas 24: 169-178, 2002.

This work is licensed under a Creative Commons Attribution-NonCommercial-NoDerivatives 4.0 International (CC BY-NC-ND 4.0) License. 